\title{
Caracterización molecular de cubios (Tropaeolum tuberosum Ruíz y Pavón) en el departamento de Boyacá
}

\author{
Molecular characterization of cubios (Tropaeolum tuberosum Ruíz and Pavón) \\ in the department of Boyacá
}

\section{Ana Cruz Morillo C. ${ }^{1}$; Yacenia Morillo C. ${ }^{2}$; Yeily Paola Tovar L ${ }^{3}$.}

\footnotetext{
${ }^{1}$ Ingeniero Agrónomo, Ph.D. Universidad Pedagógica y Tecnológica de Colombia, Tunja, Colombia, ana.morillo@uptc.edu.co.

2 Ingeniera Agrónomo, PhD. Universidad de Caldas, Manizales, Colombia, yacenia.morillo@unicaldas.edu.co.

${ }^{3}$ Ingeniero Agrónomo. Universidad Pedagógica de Colombia, Tunja, Colombia, yeily.tovar@uptc.edu.co.
}

Citar: MORILLO, A.; MORILLO, Y.; TOVAR, Y. 2016. Caracterización molecular de cubios (Tropaeolum tuberosum Ruíz y Pavón) en el departamento de Boyacá. Rev. Cienc. Agr. 33(2):32-42. doi: http://dx.doi.org/10.22267/ rcia.163302.50

Recibido: Mayo 04 de $2016 . \quad$ Aceptado: Junio 10 de 2016.

\section{RESUMEN}

El cubio (Tropaeolum tuberosum Ruiz y Pavón) es un tubérculo básico en la dieta de las comunidades andinas. Es un cultivo rústico, con buena adaptación y usos medicinales. Los agricultores de las zonas altas de Boyacá conservan cultivares de tubérculos como cubios, rubas e ibias, que hacen parte de su patrimonio cultural, sin embargo, en los últimos años, este germoplasma está siendo amenazado por la erosión genética causada por factores ambientales y antrópicos. No existen estudios de caracterización molecular de este germoplasma en Boyacá, por lo cual esta investigación se orientó hacia la identificación de la diversidad de once genotipos de cubios, procedentes de los municipios de Ventaquemada y San Pedro de Iguaque, utilizando siete marcadores Microsatélites Amplificados al Azar (RAMs). El análisis de similitud diferenció a los cultivares en dos grandes grupos, de acuerdo con características relacionadas con el tubérculo (color y forma). Los valores de heterocigosidad estimada estuvieron comprendidos entre 0,25 y 0,42 para los cebadores CGA y AG, respectivamente, lo cual es bajo si se compara con los resultados obtenidos en otras especies tuberosas. El valor promedio de heterocigosidad de la población fue de 0,35 y junto con el análisis de varianza molecular y el Fst muestran la existencia de variabilidad genética en los cultivares evaluados, la cual debe tenerse en cuenta en los programas de conservación y mejoramiento genético de los tubérculos andinos.

Palabras clave: Tropaeloaceae, microsatélites RAMs, diversidad genética, conservación, germoplasma. 


\begin{abstract}
The cubio (Tropaeolum tuberosum Ruíz and Pavón) is a basic tuber in the diet of Andean communities. It is a rustic crop, with medicinal uses. Farmers in the highlands of Boyacá department conserve tuber species such as cubios, rubas and ibias, as part of their cultural heritage; however, in recent years this germplasm has been threatened by genetic erosion due anthropogenic and environmental factors. There are not studies of molecular characterization of this germplasm in Boyacá, thus this research aims to identify the genetic diversity of eleven genotypes of cubios from Ventaquemada and San Pedro de Iguaque, using seven Random Amplified Microsatellite markers (RAMs). The similarity analysis differentiated the materials into two main groups, according to characteristics related to the tuber's color and shape. The estimated heterozygosity values ranged between 0.25 and 0.42 for CGA and AG primers, respectively, which is low when compared to the results obtained in other tuberous species. The heterozygosity average in the population was 0.35 , and together with the analysis of molecular variance and Fst show the existence of genetic variability in the cultivars evaluated, which should be considered in conservation programs and plant breeding of Andean tubers.
\end{abstract}

Key words: Tropaeloaceae, microsatellite RAMs, genetic diversity, conservation, germplasm.

\section{INTRODUCCIÓN}

El cubio (Tropaeolum tuberosum Ruiz y Pavón) también conocido como mashua en Perú y Ecuador e isaño o añu en Bolivia (Cadima, 2006) pertenece a la familia Tropaeolaceae y al género Tropaeolum, el cual incluye alrededor de 86 especies distribuidas en toda Sur América. Se reconocen dos subespecies: la cultivada T. tuberosum ssp. tuberosum y la silvestre T. tuberosum ssp. silvestre (Grau et al., 2003). Se encuentra distribuido desde Colombia hasta el norte de Argentina, entre los 2400 hasta los 4300 msnm y desde hace algún tiempo se cultiva también en algunas regiones de Nueva Zelanda y Canadá (Chirinos et al., 2007). En Perú y Bolivia, se encuentran las mayores áreas de siembra, donde generalmente se cultiva en asocio con otros tubérculos, aunque no son grandes extensiones, su cultivo no deja de ser importante, pues forma parte de la seguridad alimentaria de muchas familias campesinas en los Andes (Manrique et al., 2013). Es una planta herbácea, anual de unos 20 a $80 \mathrm{~cm}$ de altura, con tallos cilíndricos de 3 a $4 \mathrm{~mm}$ de espesor, con diversos grados de pigmentación. El color del follaje varía de amarillo-verde a verdeoscuro. La diversidad del cubio se encuentra en el color del tubérculo, forma, características de las yemas y color de la pulpa. La piel de los tubérculos varía desde el blanco hasta el violeta-púrpura muy oscuro, pasando por el amarillo, naranja, rojo y rosado; además, puede ser de un solo tono o presentar manchas y rayas contrastantes con el ápice o bajo las yemas. A diferencia de la oca y el ulluco, las flores de la mashua son profusas y en grupos que producen semillas viables con alta tasa de germinación (Clavijo et al., 2011).

El cubio es una especie rústica que puede adaptarse bien a bajas temperaturas y suelos pobres (Grau et al., 2003). Es resistente a nematodos, a insectos plaga como el gusano blanco de la papa Premnotrypes spp. Sus tubérculos tienen un elevado contenido de proteína, carbohidratos, fibra, ácido ascórbico (Vitamina C) y calorías. Contiene una elevada concentración de glucosinolatos, los cuales además de darle un sabor amargo al tubérculo le confieren propiedades antibióticas, antioxidantes insecticidas, nematicidas, anticancerígenas y diuréticas (Flores et al., 2003; Grau et al., 2003; Chirinos et al., 2008 a,b; Manrique et al., 2013).

A pesar de ser el cuarto cultivo en orden importancia en la región Andina después de la papa (Solanum tuberosum L.), la oca (Oxalis tuberosa Molina) y el 
ulluco (Ullucus tuberosus Caldas), ha sido marginalizado, pues su consumo y producción se limitan a las comunidades rurales, en el marco de una agricultura de subsistencia. Por lo tanto, es un cultivo poco conocido en el mundo y por ende poco valorado. Esta marginalización es debida principalmente a dificultades en el mercadeo del producto, bajo consumo, laboriosos procesos de cocción y bajo retorno económico (Malice et al., 2010). Sin embargo, los agricultores andinos mantienen la diversidad genética a través de las variedades locales las cuales enfrentan varias presiones de crecimiento, tales como el incremento de la población, pobreza, cambio climático, introducción de variedades modernas e integración dentro de economías nacionales de mercado. Como consecuencia de estas presiones, se observa una alarmante pérdida de variabilidad genética en las especies de tubérculos andinos (Iriondo et al., 2008; Malice et al., 2010).

Teniendo en cuenta que la conservación y caracterización de los recursos genéticos es una necesidad prioritaria para estos cultivos andinos, en el Centro Internacional de la Papa (CIP) se conservan 113 accesiones de cubios procedentes de diferentes comunidades campesinas de Perú, Bolivia y Argentina, las cuales han sido caracterizadas tanto morfológica como molecularmente (usando marcadores AFLP). Se encontró una alta diversidad genética dentro del cultivo, poniendo de manifiesto la necesidad de realizar nuevas colectas con la finalidad de estudiar y entender mejor la diversidad del cultivo (Manrique et al., 2013). Bolivia es parte de los ocho centros de biodiversidad y domesticación más importantes en el mundo y cuenta con un germoplasma de tubérculos andinos muy heterogéneo, constituido por 1200 accesiones de papa, 500 de oca, 200 de papalisa y 80 de isaño (Cadima, 2006).

En Colombia, la Universidad Nacional, cuenta con una Colección Central de cubios que está conformada por 65 accesiones de diferentes zonas del país, las cuales han sido caracterizadas fenotípica y genotípicamente (Clavijo et al., 2011) y cuantificadas en el contenido de glucosinolatos. Aguirre et al. (2012), caracterizaron el sistema de producción de tubérculos andinos en la provincia de Sugamuxi, Boyacá, encontrando que T. tuberosum fue la especie con mayor número de unidades productivas registradas (66\%). Sin embargo se evidenció que la producción y el consumo muestran una tendencia decreciente y que los tubérculos andinos no han sido objeto de mejoramiento ni de industrialización, a pesar del potencial ampliamente reconocido a nivel internacional, que permitiría superar las limitaciones actuales del cultivo (Jaramillo, 1999). La Universidad Javeriana y la Corporación para el desarrollo participativo y sostenible de los pequeños agricultores colombianos (Corporación PBA), han adelantado procesos de investigación y fomento de conservación de ibias, cubios y rubas, en pequeños sistemas productivos de Turmequé y Ventaquemada, Boyacá (Aguirre et al., 2012).

La variabilidad genética de los cubios ha sido descrita frecuentemente en términos de características morfológicas y agronómicas (Malice et al., 2010). Sin embargo, en años recientes se ha cuantificado su diversidad genética a nivel bioquímico y molecular, utilizando isoenzimas (Monteros, 1997), SRAP (Ortega et al., 2007). La técnica ISSR ha sido exitosamente usada para evaluar la diversidad genética en accesiones cultivadas de oca, ulluco y cubios (Pissard et al., 2006, 2008a, b, c; Malice et al., 2010). Estos estudios demuestran la existencia de variabilidad que debe ser conservada y cómo las prácticas de cultivo de los agricultores influencia la estructura y la diversidad genética de los tubérculos andinos.

Entre los marcadores microsatélites los RAMs (Microsatélites Amplificados al Azar) son muy útiles para medir la diversidad genética en plantas, animales y microorganismos, además permiten detectar la variación a nivel intra e inter específico, nonecesitainformación previay esuna metodología 
útil para la determinación de parámetros genéticos (Morillo et al., 2015). Teniendo en cuenta que los tubérculos andinos, y especialmente los cubios, son especies amenazadas por erosión genética, y que la colección, caracterización u evaluación de germoplasma en el Altiplano Cundiboyacense es escasa, el presente trabajo de investigación tuvo como objetivo principal, la caracterización molecular de los morfotipos de cubios en el departamento de Boyacá, usando marcadores microsátelites RAMs, con el propósito de identificar la diversidad genética.

\section{MATERIALES Y MÉTODOS}

Se evaluaron un total de once cultivares materiales de cubios, los cuales fueron colectados en el departamento de Boyacá, especialmente en los municipios de Ventaquemada y San Pedro de Iguaque en fincas de productores y ecosistemas paramunos (Tabla 1), los cuales poseen suelos francos, precipitación promedio de $870 \mathrm{~mm} / \mathrm{año}$, temperatura promedio anual de $15^{\circ} \mathrm{C}$ y una humedad relativa entre el 70 a $80 \%$.
Caracterización molecular. La caracterización molecular de los cubios se llevó a cabo en los Laboratorios de investigación en Biología Molecular de la Universidad Pedagógica y Tecnológica de Colombia, Tunja.

Para la extracción de ADN se utilizó el protocolo de Dellaporta et al. (1983). Los ADN totales se visualizaron en geles de agarosa al 0,8\%, teñidos con Gel Red en una cámara Maxicell Primo EC340 Electroforesis Gel System. Para determinar la concentración de ADN de cada accesión se hizo una curva de dilución con ADN del bacteriófago Lambda de concentración inicial 20ng/ $\mu$ l. El ADN cuantificado se diluyó en agua tipo HPLC a un volumen total de $100 \mu \mathrm{l}$ a $10 \mathrm{ng} / \mu \mathrm{l}$ y se almacenó a $-20^{\circ} \mathrm{C}$. Para el análisis RAMs se utilizaron siete cebadores sintetizados por Technologies Inc. (Tabla 2). Para la reacción de amplificación con RAMs se preparó el cóctel en un tubo estéril de microcentrífuga $(1,5 \mathrm{ml})$ para un volumen final de $25 \mu$ l. La mezcla de reacción se preparó con buffer 1X, MgCl2 1,5mM, DNTPs 0,2mM, Taq Polimerasa $1 \mathrm{U}$, cebador $2 \mu \mathrm{M}$ y ADN genómico 10ng.

Tabla 1. Cultivares de cubios [Tropaeolum tuberosum] utilizados para la caracterización molecular

\begin{tabular}{llccc}
\hline $\mathbf{N}^{\circ}$ & Procedencia & $\begin{array}{c}\text { Altura } \\
(\mathbf{m s n m})\end{array}$ & $\begin{array}{c}\text { Latitud } \\
\text { norte }\end{array}$ & $\begin{array}{c}\text { Longitud } \\
\text { oeste }\end{array}$ \\
\hline 1C & Ventaquemada & 2630 & $5,36^{\circ}$ & $73,33^{\circ}$ \\
2C & S. P de Iguaque & 2480 & $5,61^{\circ}$ & $73,52^{\circ}$ \\
3C & S.P de Iguaque & 2536 & $5,57^{\circ}$ & $73,52^{\circ}$ \\
4C & Puente de Boyacá & 2820 & $5,45^{\circ}$ & $73,48^{\circ}$ \\
5C & Ventaquemada & 2630 & $5,36^{\circ}$ & $73,21^{\circ}$ \\
6C & Ventaquemada & 2630 & $5,36^{\circ}$ & $73,46^{\circ}$ \\
7C & S.P de Iguaque & 2536 & $5,57^{\circ}$ & $73,52^{\circ}$ \\
8C & Puente de Boyacá & 2820 & $5,45^{\circ}$ & $73,21^{\circ}$ \\
9C & Ventaquemada & 2630 & $5,36^{\circ}$ & $73,52^{\circ}$ \\
10C & Ventaquemada & 2630 & $5,36^{\circ}$ & $73,52^{\circ}$ \\
11C & S.P de Iguaque & 2536 & $5,57^{\circ}$ & $73,21^{\circ}$ \\
\hline
\end{tabular}


Tabla 2. Cebadores utilizados para la caracterización molecular de cubios (Tropaeolum tuberosum R y P.).

\begin{tabular}{ll}
\hline Cebadores & Secuencia (5'a 3') \\
\hline CCA & DDB $(\mathrm{CCA})_{5}$ \\
CGA & DHB $(\mathrm{CGA})_{5}$ \\
ACA & $\mathrm{BDB}(\mathrm{ACA})_{5}$ \\
AG & $\mathrm{HBH}(\mathrm{AG})_{7} \mathrm{~A}$ \\
$\mathrm{CT}$ & $\mathrm{DYD}(\mathrm{CT})_{7} \mathrm{C}$ \\
$\mathrm{TG}$ & $\mathrm{HVH}(\mathrm{TG})_{7} \mathrm{~T}$ \\
$\mathrm{CA}$ & $\mathrm{DBDA}(\mathrm{CA})_{7}$ \\
\hline
\end{tabular}

La amplificación se llevó a cabo en un termociclador C 1000 Programmable Termal Controller (MJ. Research, Inc). La desnaturalización inicial fue a $95^{\circ} \mathrm{C}$ durante 5 minutos; desnaturalización a $95^{\circ} \mathrm{C}$ por 30 segundos, hibridación a una temperatura de $50^{\circ} \mathrm{C}$ (cebador AG y CA), $55^{\circ} \mathrm{C}$ (cebador CCA-TG-CT) y $58^{\circ} \mathrm{C}$ (cebador GT-CGA) durante 45 segundos, una extensión de $72^{\circ} \mathrm{C}$ por 2 minutos, 37 ciclos desde la desnaturalización a extensión y por último una extensión a $72^{\circ} \mathrm{C}$ durante siete minutos. Los productos de amplificación se separaron por electroforesis en geles de agarosa de alta resolución al 1,5\% a 90 voltios durante tres horas visualizándose en un transiluminador.

Análisis estadístico de la información. Se generó una matriz binaria de ausencia (cero) y presencia (uno). La similitud genética entre los individuos se calculó utilizando el coeficiente de similitud de Nei y Li (1979) también conocido como índice de DICE (Sneath y Sokal, 1973). El análisis clúster se realizó por el método UPGMA y se generó un dendrograma utilizando el paquete estadístico NTSYS (Numerical Taxónoma System for personal Computer, versión 2.02 PC). Para evaluar la diversidad genética se estimó la heterocigosidad insesgada y el porcentaje de loci polimórficos utilizando el paquete estadístico TFPGA (Tools For Population Genetic Analices), versión 1,3,
1997. Se determinó el F estadístico insesgado con un intervalo de confianza del 95\%. Se realizó el Análisis de Varianza Molecular (AMOVA) usando el programa GEnALEX 6,5.

\section{RESULTADOS Y DISCUSIÓN}

El análisis mediante el coeficiente de Nei-Li a similitud de 0,50 permitió diferenciar el germoplasma de cubios en dos grandes grupos (Figura 1). En el grupo I se encuentran las muestras que fueron colectadas en distintas fincas de Ventaquemada y San Pedro de Iguaque. Se puede observar que los agrupamientos corresponden con las características morfológicas de los materiales, siendo los cubios colectados en Ventaquemada (1C) los que menor valor de similitud mostraron con el resto. Resultados similares fueron encontrados en los estudios de caracterización morfológica de Leguizamo (Morillo et al., 2016), donde se observaron variaciones en el color de la superficie del tubérculo, color de la pulpa y en el ancho y la longitud de la lámina foliar.

En el grupo II, se muestra una distribución más laxa de los individuos procedentes de los diferentes sitios donde se realizó la colecta, poniendo de manifiesto el intercambio de material vegetal entre los agricultores. Se encontraron valores de similitud de 0,85 (7C y 11C) que muestran una alta homogeneidad lo cual puede atribuirse al sistema de propagación vegetativa de estas especies ciclo tras ciclo y a la escasa oferta de nuevos cultivares de siembra. A nivel morfológico presentaron características muy similares en cuanto al color y forma del tubérculo. Sin embargo, en este grupo el material 6C procedente de Ventaquemada, fue el que presentó un menor valor de similitud $(0,60)$ con el resto de los materiales del grupo, lo cual es importante desde el punto de vista de la variabilidad, ya que este tipo de materiales deben ser conservados porque constituyen una nueva fuente de genes para estas pobla- 


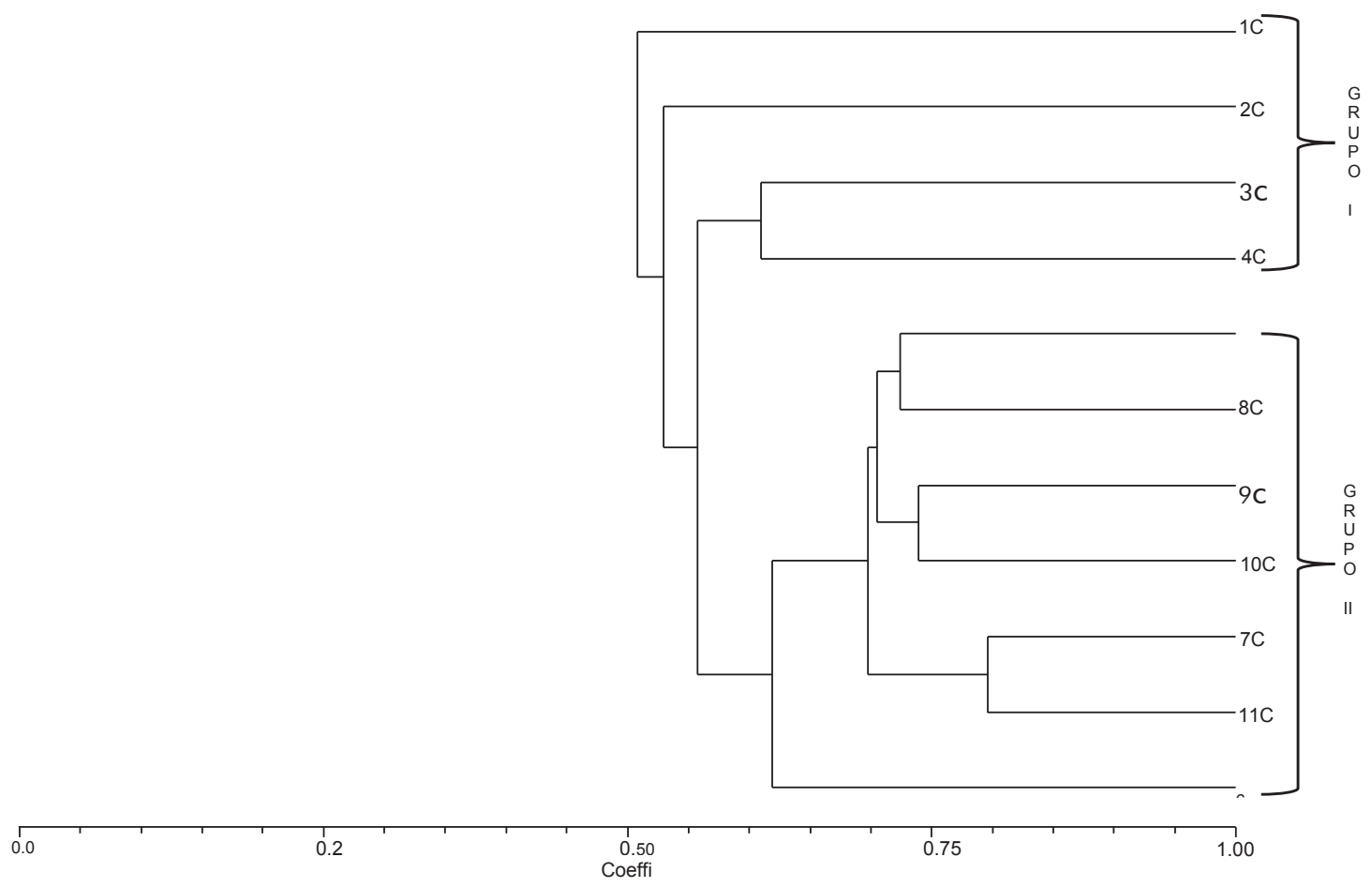

Figura 1. Dendrograma de once cultivares de cubios, Tropaeolum tuberosum, basado en el coeficiente de similitud de Nei-Li y calculado con siete marcadores RAMs, con el método de clasificación UPGMA, SAHN y TREE de NTSYs-pc Versión 1.8 [Exeter Software, Y, USA].

ciones tan homogéneas. Morillo et al. (2016) ya había reportado que este material presenta características morfológicas diferentes en cuanto a la forma, color, tamaño del tubérculo, hábito de floración entre otros.

En general, se pudo observar que los agrupamientos están fuertemente correlacionados con las características morfológicas asociadas al tubérculo. Se presentaron valores de similitud $(0,60$ $0,85)$ mucho más altos que los encontrados para la ibias (0,6; Morillo et al., 2016). Cadima (2006) ya había reportado resultados similares donde estableció que los cubios son uno de los tubérculos andinos menos variables especialmente en características relacionadas con el color y la forma del tubérculo. Las distancias genéticas en este estudio fueron menores a las encontradas en otros estudios de diversidad genética en especies de tubérculos andinos (Pissard et al., 2006; Malice et al., 2010), lo cual muestra el alto grado de homogeneidad de los cultivares y la necesidad de incrementar la variabilidad genética existente.

Los siete cebadores RAMs generaron un total de 165 bandas, de las cuales el 88\% fueron polimórficas, con pesos moleculares entre 300 y $1.300 \mathrm{~Kb}$. El número de bandas encontrado en este estudio se considera pertinente para estimar los parámetros genéticos en los materiales de cubios colectados (Malice et al., 2010; Navarro et al., 2010; Veramendi et al., 2013). El cebador TG fue el que mayor aporte hizo a la variación genética observada, con un Fst de 0,58, lo cual significa que es un marcador muy discriminante y que puede ser usado en otros estudios de diversidad genética en especies tuberosas (Tabla 3). 
Tabla 3. Valores de Heterocigosidad, Fst y Porcentaje de loci polimórficos para los cebadores RAMs evaluados en los once materiales de cubios.

\begin{tabular}{cccccc}
\hline Cebador & $\mathbf{N}^{\circ}$ Loci & $\begin{array}{c}\text { He } \\
\text { Estimada }\end{array}$ & $\begin{array}{c}\text { \% Loci } \\
\text { Polimórficos } \\
\mathbf{( 9 5 \% )}\end{array}$ & Fst & SD \\
\hline ACA & 31 & 0,33 & 87 & 0,26 & 0,07 \\
AG & 32 & 0,42 & 100 & 0,27 & 0,04 \\
CA & 18 & 0,39 & 97 & 0,15 & 0,05 \\
CCA & 37 & 0,37 & 89 & 0,38 & 0,05 \\
CT & 21 & 0,33 & 90 & 0,21 & 0,04 \\
TG & 24 & 0,30 & 83 & 0,58 & 0,04 \\
CGA & 24 & 0,25 & 71 & 0,11 & 0,02 \\
TOTAL & $\mathbf{1 6 5}$ & $\mathbf{0 , 3 5}$ & $\mathbf{8 8}$ & $\mathbf{0 , 3 0}$ & $\mathbf{0 , 0 1}$ \\
\hline
\end{tabular}

El polimorfismo observado con los RAMs varió de acuerdo con su composición y longitud de la secuencia. Algunas tendencias en general mostraron que los motivos repetidos que contenían una combinación de nucleótidos A, G o C, produjeron patrones de bandas de alta resolución y polimorfismo. Resultados similares con ISSRs fueron encontrados por Pissard et al. (2008 a) al estudiar la diversidad y la estructura genética de 68 accesiones de cubios colectados en nueve departamentos de Perú. La alta frecuencia de esos motivos (motifs) había sido reportada en otros cultivos tales como arroz (Joshi et al., 2000), papa (Bornet et al., 2002), y café (Ruas et al., 2003) entre otras. Sin embargo, los autores señalan que las repeticiones TG y GT parecen ser raras en estas especies porque obtuvieron o no pocas bandas, contrario a este estudio en donde el primer TG fue el que mayor aporte hizo a la diferenciación genética de los materiales.

Los valores de heterocigosidad estimada estuvieron comprendidos entre 0,25 para el cebador CGA y 0,442 para el AG (Tabla 3). El valor promedio para la población fue de 0,35 , con una desviación estándar de 0,01, el cual fue bajo si se compara con los resultados obtenidos por Morillo et al. (2015) quien caracterizó molecularmente diez cultivares de ibias (Oxalis tuberosa Mol.) con marcadores
RAMs, encontrando un valor de heterocigosidad de 0,39. Igual ocurrió en otros estudios de diversidad genética donde se han usado marcadores microsatélites y los valores son mucho más altos, por ejemplo, en los estudios realizados por Moscoe y Emshwiller, (2015) en Oxalis tuberosa.

Malice et al. (2010), estudiando la diversidad y la estructura genética de tres especies de tubérculos andinos oca (Oxalis tuberosa M.), ulluco (Ullucus tuberosus C.) y cubios (Tropaeolum tuberosum R. y Pav.) con marcadores ISSR, en el microcentro de Huanuco en Perú, encontraron que está última muestra una diversidad significativamente más alta que las otras dos especies evaluadas $(\mathrm{He}=0,46)$. Sin embargo, los valores de heterocigosidad estimada de los cubios evaluados son más altos que lo encontrado por Navarro et al. (2010) en la evaluación morfoagronómica y molecular de Solanum tuberosum L., y Solanum phureja procedentes del departamento de Nariño usando cinco marcadores RAMs $(\mathrm{He}=0,23)$. En 32 accesiones de ibias del Sur de América (Argentina, Bolivia, Chile y Perú), Pisssard et al. (2006) también reportaron un valor bajo de heterocigosidad $(\mathrm{He}=0,28)$ lo cual fue atribuido principalmente a la propagación vegetativa y a la estrategia de conservación de la especie. 
Los valores de heterocigosidad encontrados en este estudio muestran que existe diversidad genética en los materiales de cubios evaluados, lo cual corrobora los estudios de Morillo et al. (2016) usando marcadores morfológicos también señaló la alta variabilidad de estos materiales. A pesar de la propagación vegetativa existe variabilidad, que también se ha reportado en otras especies de tubérculos andinos, poniendo de manifiesto una subestimación de su diversidad genética en los campos (Pissard et al., 2008b; Elías et al., 2001a,b; Malice et al., 2010).

El valor de Fst promedio para los once materiales de cubios evaluados fue de 0,30, con una desviación estándar de 0,01. Según Wright (1978) valores por encima de 0,25 , muestran gran diferenciación genética. El Fst encontrado en este estudio es una variable importante, puesto que ayuda a entender la dinámica espacio-temporal de los materiales de cubios, así como la estructura de cruzamientos entre ellos, además este resultado se puede explicar por el proceso de propagación vegetativa que sufren estos materiales ciclo tras ciclo llevando a la población a la homogeneidad. En el Análisis de Varianza Molecular (AMOVA) para los dos grandes grupos formados, se encontró que el $81 \%$ de la variación total observada se debe a las diferencias aportadas por cada uno de los individuos que conforman cada uno de los grupos (Tabla 4). Esta alta variación podría indicar niveles de subdivisión mayores a los consi- derados en estudio. El 19\% restante de la variación se debe a las diferencias entre los grupos, la cual también puede ser aprovechada dentro de los programas encaminados a la conservación y mejoramiento genético de la especie. Resultados similares han sido encontrados por Pissard et al. (2008c) donde la partición del AMOVA, mostró que la mayoría de la diversidad genética está dentro de las regiones (80\%), con una proporción relativamente baja entre regiones (20\%), el cual puede ser explicado por los efectos a largo plazo de la dinámica espacio-temporal del germoplasma, además del intercambio de semilla entre los agricultores, lo cual tiende a reducir la variabilidad y a que se presente la erosión genética en la población.

En este estudio, no se pudo observar una relación directa entre la estructura genética y el sitio de colecta de los cubios, como se ha observado en otros estudios de tubérculos andinos donde existe una tendencia geográfica a la diferenciación genética a lo largo de los Andes (Sukhotu et al., 2005; Pissard et al., 2006; Pissard et al., 2008a). Lo anterior está influenciado por las fuerzas de evolución que modifican la estructura y la diversidad genética del pool genético en las especies naturales, incluyendo obviamente la recombinación sexual y quizás la mutación, seguida por una selección humana intensa para adaptación a estrés biótico y abiótico y para sus diversos usos (Pissard et al., 2008b).

Tabla 4. Análisis de Varianza Molecular (AMOVA) para los grupos formados con los cultivares de cubios (T. tuberosum R y P.) evaluados con los marcadores RAMs.

\begin{tabular}{lccccc}
\hline \multicolumn{1}{c}{ FV } & GL & SC & CM & VE & \% \\
\hline Entre Grupos & 1 & 72,69 & 72,69 & 7,68 & 19 \\
Dentro de grupos & 9 & 302,39 & 33,59 & 33,59 & 81 \\
Total & 10 & 375,09 & & 41,28 & 100 \\
\hline
\end{tabular}

$\mathrm{VE}=$ Varianza Explicada por el componente $\quad \%=$ Porcentaje de la varianza 
La diversidad genética encontrada en este estudio, indica que existe un nivel intermedio de polimorfismo en los materiales genéticos de cubios colectados en los tres municipios muestreados del departamento de Boyacá, lo cual ha sido reportado en otros estudios de diversidad genética usando otros marcadores moleculares (Okpul et al., 2005; Zhedi et al., 2005; Pissard et al., 2008 c). Por otra parte, la reproducción vegetativa y la baja importancia económica son factores que contribuyen a la pérdida de diversidad genética en los cubios, ya que produce homogeneidad en los materiales genéticos sembrados por los agricultores sin embargo, la alogamia, la presencia de formas silvestres simpátricas y los efectos de la selección humana podrían actuar a favor del incremento de esa variabilidad. Los cubios presentan formas de reproducción asexual y sexual, los cuales influencian la dinámica de la diversidad genética y su estructura en las poblaciones naturales. Sin embargo, los cubios presentan una diversidad intraespecífica más alta que la oca (Pissard et al., 2006), otro tubérculo andino de importancia económica. Esto podría deberse al hecho que los cubios pueden producir abundante semilla tanto autógama como alógama por sus sistemas de reproducción y esta última puede generar nuevos genotipos por recombinación, a las formas tradicionales de cultivo en los campos de agricultores (mezcla de variedades) y a el intercambio de material de siembra entre una región y otra (Cadima et al., 2006; Malice et al., 2010).

Los tubérculos andinos son especies nativas de las tierras altas de los Andes, donde han sido cultivadas y constituyen un alimento básico, son parte de la cultura y la expresión social de las poblaciones andinas, sin embargo, fuertemente relegadas. La diversidad fenotípica de estos cultivos es muy alta, y ha sido mantenida por comunidades locales, no obstante este germoplasma está sometido a una alta erosión genética, por lo cual se deben plantear estrategias para su manejo y conservación, además se necesita entender mejor la evolución de los cultivos clonales en los sistemas agrícolas tra- dicionales, su papel cultural, económico, social, el impacto del intercambio de semillas, la reproducción sexual en sucesivas generaciones y el rol del agricultor en los procesos de diversidad genética de estas especies.

\section{CONCLUSIONES}

Mediante la técnica RAMs se determinó que existe una alta diversidad genética de los cubios en el departamento de Boyacá en comparación con otros departamentos, la cual está muy correlacionada con los caracteres morfológicos como forma, tamaño y color del tubérculo.

En el Análisis de Varianza Molecular se encontró que las diferencias observadas en los cultivares de cubios se debe a la variación que existe en cada uno de sus individuos, lo cual sugiere niveles de subdivisión mayores a los considerados en este estudio.

La variabilidad genética en los cubios, debe ser considerada dentro de las estrategias de conservación y manejo de los recursos fitogenéticos andinos, especialmente a nivel de finca, dado que son los pequeños agricultores quienes han mantenido este recurso fitogenético por años, además que representa un alimento básico para las comunidades andinas y tiene usos potenciales que pueden mejorar su aceptación en el mercado.

\section{AGRADECIMIENTOS}

Los autores expresan sus más sinceros agradecimientos a los agricultores de las zonas de estudio por el aporte del material vegetal; a los Laboratorios de Investigación en Biología Molecular, GEBIMOL y BIOPLASMA de la Universidad Pedagógica y Tecnológica de Colombia. Al grupo de Investigación Competitividad, Innovación y Desarrollo Empresarial (CIDE). 


\section{REFERENCIAS BIBLIOGRÁFICAS}

AGUIRRE, S.; PIRANEQUE, N.; PÉREZ, I. 2012. Sistema de producción de tubérculos andinos en Boyacá, Colombia. Cuadernos de Desarrollo Rural. 9(69):257 273.

BORNET, B.; GORAGUER, F.; JOLY, G.; BRANCHARD, M. 2002 Genetic diversity in European and Argentinian cultivated pótateles (Solanum tuberosum ssp. Tuberosum) detected by inter-simple sequence repeats (ISSRs). Genome. 45(3):481 - 484. doi: 10.2225/vol16issue6-fulltext-15.

CADIMA, F. 2006. Tubérculos. In. R. Moraes, B. Ollgaard, L.P. Kvist, F. Borchsenius and H. Balslev H (ed.) Botánica Económica de los Andes Centrales. Universidad Mayor de San Andrés, La Paz, Bolivia. 347 - 369 p.

CLAVIJO, N.; COMBARIZA, J.; BARÓN, M. 2011. Recognizing rural territorial heritage: characterization of Andean tuber production systems in Boyacá. Agronomía Colombiana. 29(2):315 - 322

CHIRINOS, R.; CAMPOS, D.; COSTA, N.; ARBIZU, C.; PEDRESCHI, R.; LARONDELLE Y. 2008a. Phenolic profiles of Andean mashua (Tropaeolum tuberosum Ruíz and Pavón) tubers: Identification by HPLC-DAD and evaluation of their antioxidant activity. Food Chemistry. 106(3):1285 - 1298.

CHIRINOS, R.; CAMPOS, D.; WARNIER, M.; PEDRESCHI, R.; REES, J.; LARONDELLE, Y. 2008b. Antioxidant properties of mashua (Tropaeolum tuberosum) phenolic extracts against oxidative damage using biological in vitro assays. Food Chemistry. 111(1):98 - 105.

CHIRINOS, R.; ROGEZ, H.; CAMPOS, D.; PEDRESCHI, R.; LARONDELLE, Y. 2007. Optimization of extraction conditions of antioxidant phenolic compounds from mashua (Tropaeolum tuberosum Ruíz y Pavón) tubers. Separation and Purification Technology. 55(2):217 - 225. DELLAPORTA, S.; WOOD, J.; HICKS, J. 1983. A plant DNA minipreparation: Versión II. Plant Molecular Biology Reporter. 1 (4):19 - 21.

ELIAS, M.; PANAUD, 0.; ROBERT, T. 2001a. Traditional management of cassava morphological and genetic diversity by the Makushi Amerindians (Guyana, South America): Perspectives for on-farm conservation of crop genetic resources. Euphytica. 120(1):143 - 157. doi: 10.1023/A:1017501017031.
ELIAS, M.; PENET, L.; VINDRY, P.; MCKEY, D.; PANAUD, 0.; ROBERT, T. 2001b. Unmanaged sexual reproduction and the dynamics of genetic diversity of a vegetatively propagated crop plant, cassava (Manihot esculenta Crantz), in a traditional farming system. Molecular Ecology. 10(8):1895 - 1907. doi: 10.1046/j.09621083.2001.01331.x.

FLORES, H.; WALKER, T.; GUIMARAES, R.; BAIS, H.; VIVANCO, J. 2003. Andean root and tuber crops: Underground rainbows. Horticulture Science. 38 (2):161 $-167$.

GRAU, A.; ORTEGA, R.; NIETO, C.; Hermann, M. 2003. Mashua (Tropaeolum tuberosum Ruiz \& Pav.). Promoting the conservation and use of underutilized and neglected crops. International Potato Center, Lima. International Plant Genetic Resources Institute (IPGRI), Rome, Italy. 124 p.

IRIONDO, J.; MAXTED, N.; DULLO0, E. 2008. Conservating plant genetic diversity in protected areas: Population management of crop wild relatives. CABI Publishing, Wallingford, 144p.

JARAMILLO, L. 1999. Módulo I: Ciencia, tecnología, sociedad y desarrollo. En: Instituto Colombiano para el Fomento de la Educación Superior, ICFES (ed.). Santa Fe de Bogotá: ARF0. 145 p.

JOSHI, S.P.; GUPTA, V.S.; AGGARWAL, R.K.; RANJEKAR, P.K.; BRAR, D.S. 2000 Genetic diversity and phylogenetic relationship as revealed by intersimple sequence repeat [ISSR] polymorphism in the genus Oryza. Theoretical and Applied Genetics. 100(8):1311 -1320. doi: $10.1007 / \mathrm{s} 001220051440$.

MALICE, M.; BIZOUX, J.; BLAS, R.; BAUDOIN, J. 2010. Genetic diversity of Andean tuber crop species in the in situ microcenter of Huanuco, Perú. Crop Science. 50(5):1915 - 1923. doi:10.2135/cropsci2009.09.0476.

MANRIQUE, I.; ARBIZU, C.; VIVANCI, F.; GONZÁLEZ, R.; RAMÍREZ, C.; CHÁVEZ, O.; TAY, D.; ELÍS, D. 2013. Tropaeolum tuberosum Ruíz and Pav. Colección de germoplasma de mashua conservada en el Centro Internacional de la Papa (CIP). Primera Edición. Centro Internacional de la Papa, La Molina, Perú. 34 - 77 p.

MONTEROS, A.; MAZO, N.; CASTILLO, R. 1997. Estudio de la variación morfológica e isoenzimática de 78 entradas de mashua (Tropaeolum tuberosum) en Santa Catalina, INIAP. 34 p. 
MORILLO, A.; MORILLO, Y.; LEGUIZAMO, M. 2016. Diversidad genética de ibias Oxalis tuberosa y de cubios Tropaelum tuberosum Ruíz y Pavón en Boyacá. Temas agrios. 21 (1): 44 - 53.

MORILLO, A.; MORILLO, Y.; PINZÓN, H. 2015. Molecular characterization of the plum collection (Prunus domestica L. Borkh) of the Pedagogical and Technological University of Colombia. African Journal of Biotechnology. 14(3):257 - 263. doi: 10.5897/AJB2014.14003.

MOSCOE, L.; EMSHWILLER, E. 2015. Diversity of Oxalis tuberosa Molina: a comparison between AFLP and microsatellite markers. Genetic Resources and Corp. Evolution. 62(3): 335-347. doi: 10.1007/s10722014-0154-x.

NEI, M.; LI, W. 1979. Mathematical model for studying genetic variation in terms of restriction endonuclease. Proceedings of the National Academy Sciences. 79(10):5267 - 5273.

NAVARRO, C.; BOLAÑOS, L.; LAGOS, T. 2010. Caracterización morfoagronómica y molecular de 19 genotipos de papa guata y chaucha (Solanum tuberosum L. y Solanum phureja Juz et Buk) cultivados en el departamento de Nariño. Revista de Agronomía. 27(1):27 - 39.

OKPUL, T.; MACE, E.S.; GODWIN, I.D.; SINGH, D.; WAGIH, ME. 2005. Evaluation of variability among breeding lines and cultivars of taro (Colocasia esculenta) in Papua New Guinea using ISSR fingerprinting and agro-morphological characterization. Plant Genetics Resources Newsletter. 143(1):8 - 16.

ORTEGA, 0.; DURAN, E.; ARBIZU, R.; ROCA, W.; POTTER, D.; QUIROS, C. 2007. Pattern of genetic diversity of cultivated and non-cultivated mashua, Tropaeolum tuberosum, in the Cusco region of Perú. Genetic Resource Crop Evolution. 54 (4):807 - 821. doi: 10.1007/s10722-0069160-y.

PISSARD, A.; GHISLAIN, M.; BERTIN, P. 2006. Genetic diversity of the Andean tuber-bearing species, oca [Oxalis tuberosa Mol.], investigated by inter-simple sequence repeats. Genome. 49(1):8 - 16. Doi: 10.1007/s10709007-9150-9.

PISSARD, A.; ARBIZU, C.; GHISLAIN, M.; BERTIN, P. 2008a. Influence of the geographical provenance on the genetic structure and diversity of the vegetatively propagated Andean tuber crop, mashua (Tropaeolum tuberosum), highlighted by ISSR markers and multivariate analysis methods. International Journal. Plant Sciences. 169(9):1248 - 1260. doi: 10.1086/591979.

PISSARD, A.; ARBIZU, C.; GHISLAIN, M.; FAUX, A.; PAULET, S.; BERTIN, D. 2008b. Congruence between morphological and molecular markers inferred from the analysis of the intramorphotype genetic diversity and the spatial structure of Oxalis tuberosa. Molecular Genetics. 132(1):71 - 85.

PISSARD, A.; ROJAS, J.; FAUX, A.; PAULET, S.; BERTIN, P. 2008c. Evidence of intra-varietal genetic variability in the vegetatively propagated crop oca (Oxalis tuberosa Mol.) in the Andean traditional farming system. Plant Systematics and Evolution. 270(1-2):59 - 74. doi: 10.1007/s00606-007-0605-3.

RUAS, P.; RUAS, C.; RAMPIM, L.; CARVALHO, V.; RUAS, E.; SERA, T. 2003. Genetic relationship in Coffea species and parentage determination of interspecific hybrids using ISSR (inter-simple sequence repeats) markers. Genetics and Molecular Biology. 26(3):319 - 327. doi: 10.1590/S1415-47572003000300017.

SNEATH, P.; SOKAL, R.1973. Numerical Taxonomy: The Principles and Practice of Numerical Classification. W.H. Freeman and Co. San Francisco. 573 p.

SUKHOTU, T.; KAMIJIMA, 0.; HOSAKA, K. 2005. Genetic diversity of the Andean tetraploid cultivated potato (Solanum tuberosum L. ssp. Andigena Hawkes) evaluated by chloroplast and nuclear DNA markers. Genome. 48(1):55 - 64. Doi: 10.1139/g04-086.

VERAMENDI, S.; CADIMA, X.; GABRIEL, J. 2013. Integración molecular y morfológica para la formación de la Colección Núcleo de papa de Bolivia. Revista Latinoamericana de la Papa. 17(2): 23 - 39.

WRIGHT, S. 1978. Evolution and the genetics of populations, variability within and among natural populations, Vol 4. University of Chicago Press. Chicago. 144 p.

ZHEDI, S.; SAKKA, H.; OULD, A.; RHOUMA, A.; MARRAKCHI, M.; TRIFI, M. 2005 Molecular polymorphism and genetic relationships in a Tunisian date-palm (Phoenix dactylifera L.) collection using ISSR amplification fingerprinting. Plant Genetics Resources Newsletter. 144(1):39 - 44 . 